\title{
EJNSO
}

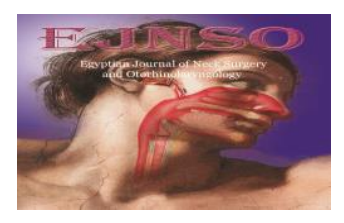

\section{Local sclerotherapy with lauromacrogol versus chemical cautery with chromic acid in treatment of idiopathic recurrent anterior epistaxis}

\author{
Marian Rafat Helmy ${ }^{1}$, Hamza El-shafaai Ahmed ${ }^{2}$ and Ahmed AbdelAleem \\ AbdelWahab ${ }^{2}$ \\ 1. Otorhinolaryngology Department El Mabara Hospital, Assiut University. \\ 2. Otorhinolaryngology, Faculty of Medicine- Assiut University.
}

\begin{abstract}
:
Introduction: Epistaxis is a very common medical problem affecting up to $60 \%$ of population at a point of their life. The classic lines of treatment of significant epistaxis include, vessel compression by packing or similar maneuvers, cauterization using various types of chemicals or heat and ligation or embolization of the bleeding vessel(s). Lauromacrogol is a relatively new compound that has been recently used as a sclerosing material in some vessel-related diseases like varicose veins and Hereditary Hemorrhagic Telengectasia.

Objectives: This study compared the efficacy of local sclerotherapy with Lauromacrogol and chemical cautery with chromic acid as treatments for recurrent idiopathic anterior epistaxis in adults.

Patients and Methods: Patients were grouped into 2 groups. In group A Lauromacrogol was injected in the submucosa. In group B Chromic acid was applied locally to the surface over the bleeding vessel(s). The primary outcome measure was bleeding control / recurrence during 3-months follow up. Secondary outcome measures were morbidity or complications and cost of both materials.

Results: The overall epistaxis control was significantly higher in group A (Lauromacrogol group) than in group B (Chromic acid group). Less morbidity was also reported in group A. Lauromacrogol was relatively more expensive.

Conclusion: Local sclerotherapy with Lauromacrogol is an effective mean for controlling recurrent idiopathic anterior epistaxis in adults. Better bleeding control with less morbidity were reported during 3-months follow up than Chromic acid cautery.
\end{abstract}

Keywords: Epistaxis, Management, Polidocanol.

\section{Introduction}

Epistaxis is one of the most common otorhinolaryngologic emergencies. It affects between $10-60 \%$ of the population during their lifetime, with $10 \%$ of them requiring medical attention $1-3$

The most common etiology of epistaxis $(80-90 \%$ of cases $)$ is idiopathic from Little's area which is an arterial plexus formed by anastomosis between terminal branches of the external and internal carotid arteries and present in the anterior septum. No clear cause can be identified in these patients. Identifiable local causes of epistaxis include nasal trauma, nasal neoplasm, iatrogenic and nasal septum abnormalities. Epistaxis can also be due to systemic factors (coagulopathy, inflammatory conditions, infectious 
diseases, vessel wall fibrosis associated with ageing, hypertension). Drugs (anticoagulant, antiplatelet, nonsteroidal anti-inflammatory and nasal sprays) may also cause epistaxis 4,5 .

Management of recurrent anterior epistaxis includes anterior nasal packing, various forms of bleeding vessel cauterization. Surgical methods like vessel ligation and embolization are necessary when the previous methods fail to control bleeding ${ }^{6}$.

Lauromacrogol (also called polidocanol) is a mixture of ethanol (5\%) and hydroxypolyethoxidodecan (95\%). It was developed as a local anesthetic at the beginning, but it was noticed that intravascular or intradermal installation produced sclerosis of nearby blood vessels. So, its use as a local anesthetic was abandoned and its use as a sclerosing material flourished ${ }^{7}$.

It has been used as a sclerosant with good results in treatment of varicose veins in the lower limbs ${ }^{7-9}$, first degree piles ${ }^{10}$, hepatic cyst ${ }^{11}$, aneurismal bone cyst ${ }^{12}$ and thyroid cysts ${ }^{13-14}$. It has also been used to treat some rare conditions like, cutaneous mucinosis 15 and lymphatic malformation ${ }^{16}$.

In 2012, Morais et al reported their 15-year experience of using lauromacrogol to treat epistaxis caused by Hereditary Hemorrhagic Telangectasia. Through submucosal and subperichondrial infiltration, they have obtained good results in controlling epistaxis in such a difficult problem ${ }^{17}$. Three years later Farneti et al reported their results comparing the efficacy of lauromacrogol infiltration versus nasal packing in the treatment of anterior epistaxis. They concluded from their study that lauromacrogol is a good alternative to nasal packing. Lauromacrogol showed them a high potential of safety, efficacy, ease of use and low cost ${ }^{18}$. There have been however some early reports of ocular complications of the use of Lauromacrogol in the area close to the eye ${ }^{19-20}$.

\section{Patients and Methods:}

This study was a prospective comparative study of the effects of sclerotherapy with Lauromacrogol versus chemical cautery using Chromic acid in management of idiopathic anterior epistaxis from Little's area. The study was conducted in the ENT department, Assiut University Hospital from April 2017 to April 2018 after obtaining approval of the institutional ethics committee No.:17101084. Date: August-2016.

Included in this study were 60 patients 18-years old or more who had recurrent idiopathic anterior epistaxis from Little's area that was marked enough to require hospital management.

Patients with epistaxis secondary to trauma, neoplasm, structural anomalies as septal spur or diseased nasal mucosa were excluded from the study. Also, patients with systemic bleeding tendency due to coagulopathy, blood vessel diseases, medications - as antiplatelets- and hypertension were excluded. For safety reasons pregnant women and patients younger than 18 years were also excluded. Patients who did not come back for the scheduled follow up were excluded too.

Before enrollment in the study a detailed general, medical, and nasal history was taken to identify the side and severity of epistaxis, and to exclude a secondary nasal or systemic cause of epistaxis. After that each patient was subjected to full general examination for the same purpose followed by local nasal examination including nasoendoscopy during or immediately after the attack of epistaxis to check the nose for site of bleeding, multiple bleeding sites, posterior bleed, diseased 
mucosa, and to better visualize the Little's area.

Patients were also subjected to the following investigations, full blood count, prothrombin time and concentration including INR, activated partial thromboplastin and platelet function to exclude bleeding tendency. Also, abdominal ultrasonography was done to exclude liver disease and early pregnancy.

Patients were grouped into 2 groups 30 patients each. Odd-number patients went to group A and even numbers to B. Both procedures were done under local anesthesia using a small piece of cotton soaked with equal volumes of lidocaine $10 \%$ solution (Astra Zeneca, RueilMalmaison, France) and epinephrine $1 / 200,000$. The former was used to achieve adequate local anesthesia for the maneuver as well as to counter any arrhythmia that may develop from the epinephrine. The later was used to decongest the area prior to the maneuver to get better visualization and to minimize the chance of bleeding during the procedure.

The procedure started shortly after the appearance of numbness over the upper lip. All procedures were done in the supine position in the operative room for the sake of safety against vaso-vagal attack.

If bleeding occurred during the maneuver in such a way that might jeopardize proper injection with Lauromacrogol or chemical cautery with chromic acid, anterior nasal packing with Vaselinized gauze was done for 2448 hours and the definite management was postponed after removal of the Vaseline pack.

In group A Lauromacrogol was used as sclerosing material. It is polyethylene glycol monododecyl ether. The material we used is commercially available as Aethoxysclerol (Kreusselpharma, Wiesbaden, Germany). It comes in 2-ml ampoules containing different concentrations of the compound. We used that containing $20 \mathrm{mg}$ of Lauromacrogol. When this material is injected in or around the vascular wall, it exerts its effect selectively on the vascular endothelium - especially the venous ones - leading to secondary thrombosis. Usually no ischemia occurs because of that venous-wall preference. The compound was put in an insulin syringe and $0.5-1 \mathrm{ml}$ was injected in the submucosa. The point of entry of the syringe was just posterior to the mucocutaneous junction opposite the bleeding vessel $\{s\}$.

Sufficient and correct injection was identified by blanching of the nasal mucosa and disappearance of the vessels in the created edema. Multiple injections might be required to achieve such blanching. Over injection should be avoided so not to obstruct the nose or dissect the mucosa from the cartilage posteriorly in such a way like what happens during injection for septal surgery.

In group B, Chromic acid $\left(\mathrm{Cr} \mathrm{H}_{2} \mathrm{O}\right.$ 4) was the material we used for chemical cautery. It is available in the form of dark brown scales in glass bottles of variable volumes (M.M.Egypt Co.). The scales may be used directly over the bleeding vessel or used as a solution after dissolving the scales in saline or water. Application of this chemical over the bleeding vessels in Little's area causes coagulative necrosis of these vessels. We used the solution in our work. A small cotton-tipped applicator was put in the chromic acid solution and transferred to the anaesthetized Little's area after opening the nose with a suitable-sized speculum. The cauterized vessels were identified by the brownish color they get. Care should be given to avoid too much saturation of the cotton tip with this chemical which might result in squeezing and spread around 
wide area. This should prevent undue cautery to the nasal vestibule, turbinate, posterior septum and even the upper lip.

A small film of panthenol cream was applied over the cauterized area to promote healing and to counter the burning effect of this caustic which usually appears after fading of the local anesthetic effect. The patients were advised to keep applying this panthenol film twice a day for 5-7 days until the formed crust separates. Patients were also advised not to self-remove the crust -which is sometimes itchy- until it separates spontaneously. Patients also were instructed to avoid forced nose blowing for the same period.

In both groups' patients were allowed to go home few minutes after the procedures. They were asked to come for follow up at 3rd day, 1 month and at 3 months. Also, patients were asked to report any morbidity or mishap by phone at any time shall any complications or new symptoms were noticed.

At the 3rd day: Both groups were evaluated for recurrence of anterior epistaxis, nasal obstruction, pain or headache, crustations, and any other new symptoms.

At one month: Both groups were also evaluated for efficacy of the procedure in controlling epistaxis as well as complications like septal perforation, adhesions, crustations, etc.

At 3rd month: Patients in both groups were evaluated for the previously mentioned complications, new symptoms and recurrence of anterior epistaxis.

The cost of both procedures was calculated (excluding costs common for both procedures). We also reported the convenience of both procedures.

\section{$\underline{\text { Statistical analysis : }}$}

The collected data were revised, organized, tabulated and statistically analyzed using statistical package for social sciences (SPSS) version 23.0 for windows. Data were presented as the Mean \pm standard deviation (SD), and percentage. Categorical variables were compared using the Chi-square $(\chi 2)$ test.

The level of significance was accepted if the $\mathrm{P}$ value was $<0.05$.

\section{Results:}

In group A, (Lauromacrogol group) there were 18 males and 12 females. Their ages ranged from 18 to 35 years with a mean \pm SD $(22.14 \pm 4.97)$.

In group $\mathrm{B}$, (Chromic acid group) there were 19 males and 11 females. Their ages ranged from 18-50 years with a mean \pm SD $(23.8 \pm 8.09)$.

The overall recurrence rate of epistaxis was significantly lower in group A than in group B table (1).

In group $A$, there was only one case of mild recurrent epistaxis. It occurred at the 5th day after injection and it was self-controlled at home by few-minutes compression over an oxymetazolinecontaining piece of cotton.

In group B there were 7 cases of recurrent epistaxis. In three cases, epistaxis was in the 2 nd day, 2 cases at the 5 th and 2 cases at 6 th day. Four cases (those with 2 nd day and another one with 5thdayepistaxis) had trivial form of bleeding that was selfcontrolled at home by the same previously mentioned compression. In 3 cases-however- bleeding was marked and necessitated coming back to the hospital and nasal packing. Table (1) showed the overall recurrence of epistaxis by the end of the study.

Table 1: Overall recurrence of epistaxis by the end of the study 


\begin{tabular}{|c|c|c|c|c|c|}
\hline \multirow{2}{*}{ Recurrence } & \multicolumn{2}{|c|}{$\begin{array}{c}\text { Group A } \\
(\mathbf{n}=\mathbf{3 0})\end{array}$} & \multicolumn{2}{|c|}{$\begin{array}{c}\text { Group B } \\
(n=30)\end{array}$} & \multirow{2}{*}{$\begin{array}{c}P . \\
\text { value }\end{array}$} \\
\hline & No. & $\%$ & No. & $\%$ & \\
\hline $\begin{array}{c}\text { No } \\
\text { recurrence }\end{array}$ & 29 & 96.7 & 23 & 76.7 & \multirow{2}{*}{0.023} \\
\hline Recurrence & 1 & 3.3 & 7 & 23.3 & \\
\hline
\end{tabular}

Apart from epistaxis, patients also reported some symptoms and morbidities during this study (table 2). Pain and nasal-crust formation were the most frequently encountered problems. In group $\mathrm{B}$, pain and crustation were reported in 14 and 13 cases respectively. Both symptoms were tolerably mild and persisted for few days. No pain or crust formation was reported in Group A. Such a difference was statistically significant.

Nasal obstruction was reported in 9 cases (3 in group A and 6 in group B.) It faded faster in group A than B (2 days versus 7 days in average). The difference was insignificant.

Sense of nasal dryness was reported in 2 cases in group A. It was persistent for 5 days. Sense of dryness was not reported from group B patients.

Table 2: Patient complains during follow up.

\begin{tabular}{|c|c|c|c|c|c|}
\hline \multirow{2}{*}{ Symptom } & \multicolumn{2}{|c|}{ Group A } & \multicolumn{2}{c|}{ Group B } & \multirow{2}{*}{ P value } \\
\cline { 2 - 5 } & No. & $\%$ & No. & $\%$ & \\
\hline Dryness & 2 & 6.7 & 0 & 0.0 & 0.469 \\
\hline Obstruction & 3 & $\begin{array}{c}10 . \\
0\end{array}$ & 6 & 20.0 & 0.470 \\
\hline Crustations & 0 & 0.0 & 13 & 43.3 & $<0.001 * *$ \\
\hline Pain & 0 & 0.0 & 14 & 46.7 & $<0.001 * *$ \\
\hline
\end{tabular}

We have not seen major complications like septal perforation, adhesions or ocular complication in any patient in either group.

When comparing the cost of both procedures, One-side injection with Lauromacrogol costs around 200-250
Egyptian pounds for the material only. This is relatively costy when compared with the very cheap Chromic acid.

Both procedures were generally simple and easy, but we experienced more convenience when doing the surface cauterization with Chromic acid than the needle injection of Lauromacrogol. Patients hesitated more on seeing the needle coming close.

\section{Discussion :}

Epistaxis is one of the most common ENT emergencies worldwide constituting around $12 \%$ of them in one study ${ }^{18}$.

Management of epistaxis has 3 integrated cornerstones. They are stopping the bleeding, to save life, identification of the site of bleeding for precise targeting that site and identification and treating the cause to prevent recurrence. The majority of epistaxis however is idiopathic from Little's area located in the anterior septum.

Lauromacrogol is a sclerosing material that has been tried to treat Hereditary Hemorrhagic Telangectasia with encouraging results ${ }^{17}$.

In this study we compared the results of 2 methods of controlling recurrent idiopathic anterior epistaxis from Little's area; local sclerotherapy with Lauromacogol which is a relatively new material in this field and surface chemical cautery using Chromic acid which is a commonly used material in Egypt since long time.

The overall success rate of epistaxis control was significantly higher in Lauromacrogol group (97\%) than Chromic acid group (77\%). Premature separation of the crust over the cauterized area with subsequent rebleeding was the most likely cause of higher re-bleeding with Chromic acid. 
Farneti et al in 2016 reported 89\% success rate of epistaxis control using submucosal Lauromacrogol injection. The better result we had may be due to the fact that we excluded while they included hypertensive patients and patients on anticoagulants ${ }^{18}$.

Some degree of nasal obstruction was present for few days with both techniques. The duration of nasal obstruction was shorter in Lauromacrogol group. The cause of nasal obstruction was different in both groups. Mucosal edema from the injected Lauromacrogol was the cause in group A while the nasal crust formed over the cauterized area was the cause in group B. Pain and nasal crust formation were not noticed in Lauromacrogol group despite being a significant problem in Chromic acid group. Being a local anesthetic and its injection was submucosal; Lauromacrogol group did not have these 2 symptoms.

\section{Conclusion:}

Sclerotherapy with Lauromacrogol is a safe and effective way for controlling anterior epistaxis from Little's area. Better control of epistaxis with less morbidity than Chromic acid cautery are to be expected. Longer follow up on larger number of patients is however required to judge better on the long-term efficacy and safety of Lauromacrogol.

Declaration of interest: The authors declared no potential conflicts of interest with respect to the research, authorship and/or publication of this article.

\section{Financial support and} sponsorship: No financial support was obtained from any source.

\section{Reference:}

1. Beck R, Sorge M, Schneider A, Dietz A. Current approaches to epistaxis treatment in primary and secondary care. Deutsches Arzteblatt international. 2018;115(2):12-22.

2. Traboulsi H, Alam E, Hadi U. Changing Trends in the Management of Epistaxis. Int $\mathrm{J}$ Otolaryngol. 2015; 2015:263987:6.

3. Ha JF, Hodge JC, Lewis R. Comparison nasendoscopic-assisted cautery versus packing for the treatment of epistaxis. ANZ J Surg. 2011;81(5):336-9.

4. Tikka T. The Aetiology and Management of Epistaxis.Otolaryngology Online Journal. 2016;6(2)

5. Viehweg TL, Roberson JB, Hudson JW. Epistaxis: diagnosis and treatment. J Oral Maxillofac Surg. 2006;64(3):511-8.

6. Carey B, Sheahan P. Aetiological profile and treatment outcomes of epistaxis at a major teaching hospital: a review of 721 cases. Ir J Med Sci. 2017;187(3)761-6.

7. Zhang J, Jing Z, Schliephake DE, Otto J, Malo, f GM , Gu YQ. Efficacy and safety of Aethoxysklerol(R) (polidocanol) $0.5 \%, 1 \%$ and $3 \%$ in comparison with placebo solution for the treatment of varicose veins of the lower extremities in Chinese patients (ESA-China Study). Phlebology. 2012;27(4):184-90.

8. King JT, O'Byrne M, Vasquez M, Wright D, Group V-I. Treatment of Truncal Incompetence and Varicose Veins with a Single Administration of a New Polidocanol Endovenous Microfoam Preparation Improves Symptoms and Appearance .Eur J 
Vasc Endovasc Surg. 2015;50(6):784-93.

9. Kurnicki J, Oseka M, Tworus R, Galazka Z. Ultrasound-guided foam sclerotherapy of great saphenous vein with $2 \%$ polidocanol - oneyear follow-up results. Wideochir Inne Tech Maloinwazyjne. 2016;11(2):67-75.

10. MoserKH, Mosch C, Walgenbach M, Bussen DG, Kirsch J, Joos AK, et al. Efficacy and safety of sclerotherapy with polidocanol foam in comparison with fluid sclerosant in the treatment of first grade haemorrhoidal disease: a randomised, controlled, singleblind, multicentre trial. Int $\mathrm{J}$ Colorectal Dis. 2013;28(10):143947.

11. Spârchez1 Z, Radu P, Zaharie1 F, Hajjar NA, Spârchez M. Percutaneous treatment of symptomatic non-parasitic hepatic cysts. Initial experience with singlesession sclerotherapy with polidocanol. Med Ultrason. 2014;16(3)222-8.

12. Mohan R, Sreekumaran GT. Secondary Aneurysmal Bone Cyst of the Scapula Treated by CT Guided Percutaneous Polidocanol Injection - A Case Report. J Clin Diagn Res. 2016;10(12): 4-6.

13. Gong X, Zhou Q, Wang F, Wu W, Chen X. Efficacy and Safety of Ultrasound-Guided Percutaneous PolidocanolSclerotherapy in Benign Cystic Thyroid Nodules: Preliminary Results. Int J Endocrinol. 2017;2017: 1-5.

14. Gong X, Wang F, Du H, Chen X, Shi B. Comparison of UltrasoundGuided Percutaneous Polidocanol Injection Versus Percutaneous Ethanol Injection for Treatment of Benign Cystic Thyroid Nodules. J Ultrasound Med. 2018(6)1423-9.
15. Juhl ME, Sidiropoulos M, Antonijevic S, Lyon M, Krunic A. Focal cutaneous mucinosis after knee replacement: A rare entity successfully treated with intralesionalpolidocanol. JAAD Case Rep. 2017;(1) 3:16-8.

16. Yamaki T, Sasaki Y, Hasegawa Y, Osada A, Konoeda H, Hamahata A, et al. Percutaneous ultrasoundguided sclerotherapy with polidocanolmicrofoam for lymphatic malformations.Journal of vascular surgery Venousand lymphatic disorders. 2017;5(5):70714.

17. Morais D, Millas T, Zarrabeitia R, Botella LM, Almaraz A. Local sclerotherapy with polidocanol (Aethoxysklerol(R)) for the treatment of Epistaxis in RenduOsler-Weber or Hereditary Hemorrhagic Telangiectasia (HHT :( 15 years of experience. Rhinology. 2012;50 (1):80-6.

18. Farneti P, Pasquini E, Sciarretta V, Macri G, Gramellini G, Pirodda A. Comparison of Local SclerotherapyWith Lauromacrogol Versus Nasal Packing in the Treatment of Anterior Epistaxis. ClinExpOtorhinolaryngol. 2016;9(2):131-5.

19. Breuninger H. [Unilateral blindness following injection of aethoxysklerol into the nasal septum with medico-legal consequences]. 1983;31(7):233-5.

20. Chamot L, Zografos L, Micheli JL Ocular and orbital complications after sclerosing injections in a case of frontal cutaneous angioma. Ophthalmologica. 1981; 182(4):193-8. doi: 10.1159 000309113. PMID:7290577. 